\title{
A psychophysiological comparison of on-sight lead and top rope ascents in advanced rock climbers
}

\author{
S. Fryer ${ }^{1}$, T. Dickson ${ }^{1}$, N. Draper ${ }^{1}$, G. Blackwell ${ }^{1}$, S. Hillier ${ }^{2}$ \\ ${ }^{1}$ School of Sciences and Physical Education, University of Canterbury, Christchurch, New Zealand, ${ }^{2}$ Tauranga Hospital, Emergency \\ Medicine, Bay of Plenty District Health Board, Tauranga, New Zealand \\ Corresponding author: Simon Fryer, University of Canterbury, Dovedale Road, Christchurch 8081, Canterbury, New Zealand. Tel: \\ +6433642987 Ext 43225, Fax: +64 3 3458131, E-mail: simon.fryer@pg.canterbury.ac.nz
}

Accepted for publication 28 November 2011

Research suggests that lead climbing is both physiologically and psychologically more stressful than top rope climbing for intermediate performers. This observation may not be true for advanced climbers, who train regularly on lead routes and are accustomed to leader falls. The aim of this study was to compare the psychophysiological stresses of lead and top rope on-sight ascents in advanced rock climbers. Twenty-one climbers (18 men and three women) ascended routes near or at the best of their ability (22 Ewbank). Psychological stress was measured preclimb using the Revised Comparative State Anxiety Inventory (CSAI-2R). Plasma cortisol was sampled at six intervals. The volume of oxygen $\left(\mathrm{VO}_{2}\right)$ and heart rate $(\mathrm{Hr})$ were measured throughout the climbs. No significant differences were found in self-confidence, somatic, or cognitive anxiety between the conditions lead and top rope. No significant differences in plasma cortisol concentration were found between any time points. No significant relationships were found between cortisol and any CSAI-2R measures. No significant differences were found between conditions for $\mathrm{VO}_{2}$ or blood lactate concentration. During the lead climb, $\mathrm{Hr}$ was significantly elevated during the last part of the route. Findings suggest that advanced rock climbers do not find lead climbing to be more stressful than top rope climbing during an on-sight ascent.
During recent years, a surge in physiological, psychological, biomechanical, and anthropometrical research has followed the increased participation of rock climbing (Mermier et al., 2000; Watts et al., 2000; NoÈ et al., 2001; Quaine et al., 2003; de Geus et al., 2006; Giles et al., 2006; Vigouroux et al., 2006; Bertuzzi et al., 2007; Hardy \& Hutchinson, 2007; Sanchez et al., 2010). Initial studies that sought to investigate the physiology of rock climbing did so by focusing on energy systems contribution, heart rate $(\mathrm{Hr})$ and volume of oxygen $\left(\mathrm{VO}_{2}\right)$ responses, blood lactate values, and energy expenditure (Billat et al., 1995; Mermier et al., 1997; Watts \& Drobish, 1998; Booth et al., 1999). Much of this research was conducted with the use of artificial climbing walls and treadwalls, and often involved the manipulation of ascent style and grade of climb with respect to the climber's experience or ability (Watts \& Drobish, 1998; Watts et al., 2000; EspaÒa-Romero et al., 2009). Billat et al. (1995) found that rock climbing requires mainly activation of the anaerobic energy systems, whereas Sheel (2004) suggested an increased reliance on aerobic metabolism. Attempts to quantify energy system contribution still remain discordant within the sport (Bertuzzi et al., 2007; Sherk et al., 2010).
It has since been suggested that rock climbing is a multidimensional activity incorporating both physiological and psychological components (Goddard \& Neumann, 1994; Draper et al., 2010). Because of the recognized psychological aspect of rock climbing, intermediate performers have begun to receive much attention (Draper et al., 2008, 2010; Hodgson et al., 2009). A small number of authors have attempted to assess the psychophysiological stress thought to be inherent within rock climbing (Williams et al., 1978; Draper et al., 2008, 2010; Hodgson et al., 2009; Sherk et al., 2010). Methods have tended to incorporate different ascent styles of lead, top rope, continuous, and on-sight climbing in order to manipulate the levels of psychophysiological stress placed on the intermediate climber.

Hodgson et al. (2009) investigated the psychophysiological responses of 12 intermediate climbers ascending under three different conditions. Ascents were designed to evoke a low (top rope), moderate (top rope and lead), and high (lead) physical and mental stress. Anxiety and self-confidence were assessed immediately postclimb using a Revised Comparative State Anxiety Inventory (CSAI-2R). Plasma cortisol samples were collected preand postclimb and were used as psychophysiological 


\section{Fryer et al.}

markers of stress. Analysis showed somatic anxiety was elevated in higher stress conditions while self-confidence decreased. Cortisol concentration was found to have a cubic relationship with self-confidence, cognitive, and somatic anxiety. Findings suggested that intermediate climbers found lead climbing to be more stressful than top roping, both physiologically and psychologically. Draper et al. (2008) studied the physiological and psychological demands of an on-sight lead in comparison with a subsequent lead on intermediate rock climbers. Significant differences were reported for preclimb cognitive and somatic anxieties as well as postclimb lactate concentration. It was suggested that the increased physiological responses were a direct reflection of the higher level of anxiety induced by an on-sight climb.

Recent psychological research into rock climbers has shown that intermediate performers find lead climbing more stressful than top rope ascents. This increase in psychological stress has been paralleled with increases in physiological stress. However, no known previous research has investigated the psychophysiological responses of more advanced rock climbers during varying styles of ascent. In view of the lack of research into advanced rock climbers, the aim of the current study was to determine whether a lead climb is physiologically and psychologically more demanding than a top rope climb.

\section{Method}

\section{Participants}

Twenty-one advanced rock climbers (18 men and three women) volunteered to take part in the current study. In accordance with Draper et al., (2011a), climbers were classified as advanced because of their self-reported best red-point and on-sight grades being 23-25 (Ewbank) and 21-22 (Ewbank), respectively. As participants were climbing near or at their best on-sight grade, three participants fell during the trial. All data from these participants were excluded from the study. All participants completed a medical health history questionnaire and gave informed consent prior to taking part in the study. Ethical approval was granted from the University of Canterbury's human ethics committee before research began.

\section{Procedure}

All participants attended three testing sessions, one in an exercise physiology laboratory and two at an indoor climbing gym (Sheer Adventure, Christchurch, NZ). All testing sessions were separated by no less than $48 \mathrm{~h}$ and no more then 10 days. For each session, participants were asked not to consume alcohol or caffeine for at least $2 \mathrm{~h}$ prior to testing. Individuals were asked not to alter their training regime in the days leading up to each session. Both lead and top rope ascents were performed on exactly the same route.

Session one consisted of anthropometrical measures (Table 1) and a treadmill (Woodway®, Waukesha, WI, USA) based on $\mathrm{VO}_{2 \max }$ using the athlete-led protocol (Draper \& Hodgson, 2008). Oxygen uptake was measured using a portable metalizer $\left(\mathrm{K}_{4} \mathrm{~b}^{2}\right.$, Cosmed, Rome, Italy) and data were averaged at 15 -s intervals. No significant differences were observed between the group's lead and top rope for any measures within Table 1 . Session two was for familiarization purposes wherein participants ascended selfselected routes wearing the $\mathrm{K} 4 \mathrm{~b}^{2}$ portable metalizer.
Table 1. Anthropometrical and physiological (mean \pm SD) data for advanced climbers

\begin{tabular}{lcc}
\hline & Top rope climb & \multicolumn{1}{c}{ Lead climb } \\
\hline Lead experience (years) & $4.3 \pm 2.1$ & $6.6 \pm 4.0$ \\
Climbs per week & $3.2 \pm 0.9$ & $3.4 \pm 1.0$ \\
Best red-point grade (Ewbank) & $25.8 \pm 1.3$ & $26.1 \pm 0.4$ \\
Best on-sight grade (Ewbank) & $22.8 \pm 0.3$ & $23.5 \pm 0.8$ \\
Age (years) & $26.25 \pm 7.3$ & $25.3 \pm 9.1$ \\
Height $(\mathrm{cm})$ & $176.0 \pm 6.5$ & $175.1 \pm 8.9$ \\
Weight $(\mathrm{kg})$ & $68.2 \pm 9.7$ & $67.8 \pm 10.4$ \\
$\mathrm{VO}_{2 \max }(\mathrm{mL} / \mathrm{kg} / \mathrm{min})$ & $60.2 \pm 8.5$ & $52.0 \pm 9.5$ \\
$\mathrm{VO}_{\max } \mathrm{Hr}(\mathrm{bts} / \mathrm{min})$ & $189.3 \pm 10$ & $193 \pm 10.7$ \\
\hline
\end{tabular}

Note: $\mathrm{SD}$, standard deviation; $\mathrm{VO}_{2}$, volume of oxygen; $\mathrm{Hr}$, heart rate.

The third and final session took place at the climbing gym. On arrival, a capillary blood sample was taken for later analyses of cortisol concentration. The participant was then informed of the grade (22 Ewbank) and his or her ascent style (lead or top rope). Ascent style was decided using a randomized design (after matching for gender, age, height, and weight). Climbers completed a specific warm-up consisting of a 5-min light jogging at $60 \%$ of $\mathrm{Hr}$ max, a warm up climb of their choice (<18 Ewbank), and 5 min of stretching and mobilizing. Following the warm-up, a second capillary blood sample was collected (15 min postarrival). The climber was then fitted with the $\mathrm{K} 4 \mathrm{~b}^{2}$ and given time to inspect the route. Before attaching themselves to the rope, blood lactate concentration was sampled along with capillary blood (preclimb (30 min postarrival)). Once the climber was attached to the rope, they filled in a CSAI-2R questionnaire (Cox et al., 2003) to measure cognitive and somatic anxiety and self-confidence. During the climbing trial, $\mathrm{Hr}$ and oxygen consumption were recorded continuously. After completing the climb, a NASA Task Load Index (Hart \& Staveland, 1988) questionnaire was completed. Blood lactate and capillary blood were sampled immediately on contact with the ground. A 30-min recovery period was started during which blood lactate concentration was sampled at 5, 10 , and $15 \mathrm{~min}$ postclimb. Capillary blood samples were also collected at 15 and $30 \mathrm{~min}$ postclimb.

\section{Blood sampling and analysis}

All capillary blood samples were taken from the first (big) toe in order to minimize the effect on climbing performance. The toe has been shown to be a reliable and alternate sampling site for the concentration of lactate in rock climbers (Fryer et al., 2011). The site was prepared using a nonalcoholic medical wipe (TYCO Healthcare, Hampshire, UK) before a Haemolance Plus (Haemedic, Ozorkow, Poland) was used to puncture the site to a depth of $1.6 \mathrm{~mm}$. Blood lactate concentration was analyzed using the portable Lactate Pro (Arkray Inc, Kyoto, Japan) device. Blood samples $(300 \mu \mathrm{L})$ for cortisol analysis were collected in a lithium heparin CB300LH Microvette (Sarstedt Aktiengesellschaft \& Co, Nümbrecht, Germany) before being immediately spun in a cr2000 centrifuge (Centurion Scientific, West Sussex, England) at $10000 \mathrm{rpm}$ for $10 \mathrm{~min}$. Once the plasma was separated, samples were stored in Eppendorf microtubes (Sarstedt Aktiengesellschaft \& Co) at $-20{ }^{\circ} \mathrm{C}$ for subsequent analysis. The plasma was analyzed for cortisol concentration using an enzyme-linked immunosorbent assay Kit (Department of Clinical Biochemistry, Christchurch Hospital, Christchurch, New Zealand) as described and validated by (Lewis \& Elder, 1985). All standards, controls, and samples were analyzed in duplicate. Intra-assay coefficients of variation were $<10 \%$. Cortisol concentration was expressed $\mathrm{nmol} / \mathrm{L}$ and were subsequently converted to $\mu \mathrm{g} / \mathrm{dL}$ and $\mathrm{ng} / \mathrm{mL}$ with a factor of 27.59 (Volovitz et al., 1995). 


\section{A psychophysiological comparison of advanced rock climbers}

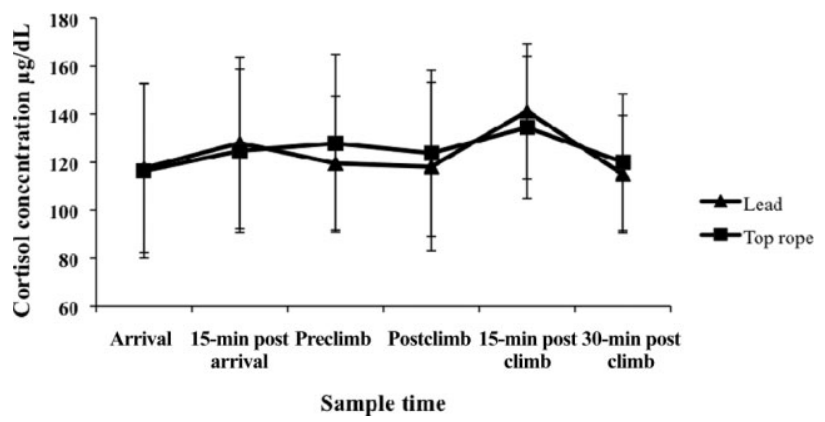

Fig. 1. Mean cortisol concentration $(\mu \mathrm{g} / \mathrm{dL})$ for lead and top rope throughout the climbing trial.

\section{Statistical analysis}

All analyses were performed using Statistical Package for the Social Sciences (SPSS) (version 19.0, Chicargo, IL, USA). All variables were assessed for normality of distribution using the one-sample Kolomogorov-Smirnov goodness-of-fit test. In order to assess differences in blood lactate, $\mathrm{VO}_{2}, \mathrm{Hr}$, and cortisol concentration between the groups lead and top rope, a series of twoway repeated measures of analysis of variance (ANOVA) was used. Where significant differences were found, two-tailed independent samples $t$-tests (with Bonferroni correction error) were calculated. Linear regression analyses were performed between all psychological measures (CSAI-2R) and cortisol concentrations. Critical $\alpha$ level was set at 0.05 for all analyses.

\section{Results}

Eighteen participants completed the current study (nine top rope and nine lead). Mean \pm SD for climb time top rope and lead was $109.89 \pm 14.65 \mathrm{~s}$ and $163.67 \pm 49.24 \mathrm{~s}$, respectively, suggesting that for on-sight climbing, a lead ascent takes significantly longer than top rope.

CSAI-2R showed a non significant difference between the ascent styles lead and top rope. Mean results suggest that there was minimal increases in cognitive $(+0.8)$ and somatic $(+0.7)$ anxiety as well as self-confidence $(+1.2)$ during the lead ascent compared with top rope. Furthermore, regression analyses revealed no significant relationships between CSAI-2R measures and plasma cortisol concentration. Figure 1 shows cortisol concentration profiles for both ascent styles. Mean values upon arrival were the same for both lead and top rope. Both ascent styles showed a peak spike at 15 min postclimb with the lead values being elevated slightly above top rope; however, this difference was shown to be non significant. After $30 \mathrm{~min}$ of passive recovery, cortisol concentration for both lead and top rope had returned to the values seen on arrival.

To further understand the differences between top rope and lead climbing, Figs 2 and 3 were produced to examine key physiological variables $\left(\mathrm{Hr}\right.$ and $\left.\mathrm{VO}_{2}\right)$ associated with each ascent style. To further break down the profiles of $\mathrm{Hr}$ and $\mathrm{VO}_{2}$, results have been displayed throughout the climb (time points are displayed as each

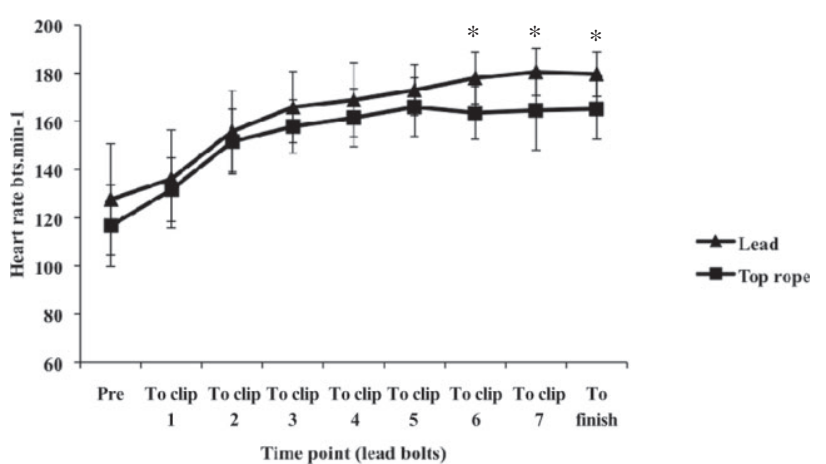

Fig. 2. Mean heart rates averaged between clips for top rope and lead climbs $(* P<0.05)$.

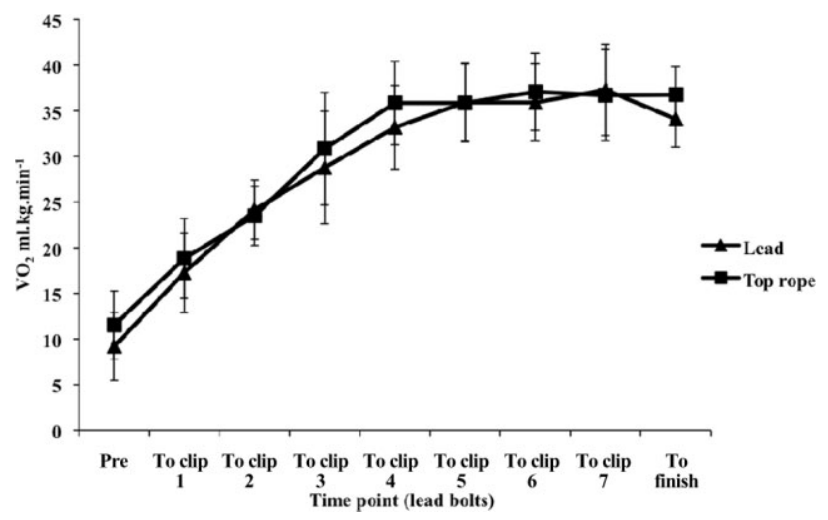

Fig. 3. Mean $\mathrm{VO}_{2}$ averaged between clips for top rope and lead climbs.

lead clip). Results for mean Hr top rope and lead represent $83.29 \%$ and $77.77 \%$ of values reached during the $\mathrm{VO}_{2 \max }$ trial. A two-way repeated measures ANOVA revealed a significant difference $\left(F_{(7)}=2.446, P=0.024\right)$ between lead and top rope throughout the climb. A series of post hoc independent sample $t$-tests revealed that $\mathrm{Hr}$ values did not significantly differ between groups from the start to clip 5. At clip 6, lead $\mathrm{Hr}$ values rose significantly above top rope and remained elevated until the finish.

An independent samples $t$-test revealed no significant differences in $\mathrm{VO}_{2 \max }$ between the groups lead and top rope. A two-way repeated measures ANOVA showed no significant differences in $\mathrm{VO}_{2}$ throughout the climb between the groups top rope and lead. Mean $\mathrm{VO}_{2}$ during top rope and lead climbing represents $55.32 \%$ and $63.88 \%$ of values attained during the $\mathrm{VO}_{2 \max }$ trial. Figure 3 represents the $\mathrm{VO}_{2}$ profile of both lead and top rope ascents throughout the climbing trial. $\mathrm{VO}_{2}$ increases linearly during both ascent styles between preclimb and clip 4. After ascending to clip $4, \mathrm{VO}_{2}$ values plateau through clips 5, 6, and 7 until the finish where lead $\mathrm{VO}_{2}$ falls slightly below top rope values.

Blood lactate concentration (Table 2) was similar preclimb for both top rope and lead. A two-way repeated measures ANOVA revealed no significant differences in 


\section{Fryer et al.}

Table 2. Mean \pm SD blood lactate concentration ( $\mathrm{mmol} / \mathrm{L})$ pre and postclimb for lead and top rope

\begin{tabular}{lll}
\hline Lactate concentration $(\mathrm{mmol} / \mathrm{L})$ & Top rope climb & Lead climb \\
\hline Preclimb & $2.3 \pm 0.7$ & $2.1 \pm 0.4$ \\
Immediately postclimb & $4.8 \pm 0.8$ & $5.2 \pm 1.1$ \\
5 min postclimb & $4.2 \pm 1.1$ & $4.5 \pm 1.3$ \\
10 min postclimb & $3.5 \pm 1.2$ & $3.7 \pm 1.2$ \\
15 min postclimb & $3.1 \pm 1.0$ & $2.9 \pm 1.0$
\end{tabular}

Note: SD, standard deviation.

blood lactate concentration during postclimb recovery after top rope and lead ascents. During $15 \mathrm{~min}$ of passive recovery, blood lactate concentration for top rope and lead decreased at similar rates. After 15-min recovery, values remained slightly elevated above those seen immediately preclimb for both ascent styles.

\section{Discussion}

All participants in this study were categorized as advanced-level climbers as per the classification criteria set out by Draper et al. (2011a). This is based on redpoint and on-sight ability grade. In addition, the physiological and anthropometrical characteristics of the climbers were similar to those found in previous studies conducted with advanced climbers (Billat et al., 1995; Booth et al., 1999; Sheel, 2003) with $\mathrm{VO}_{2 \max }$ indicating a high level of physical fitness.

In order to assess psychological anxiety among both lead and top rope conditions, participants completed the CSAI-2R immediately prior to climbing. Findings indicated that there were no significant differences in levels of cognitive and somatic anxiety or self-confidence. These findings are contrary to those of Hodgson et al. (2009), who reported significant differences between top rope and lead climbing for somatic anxiety and selfconfidence in intermediate climbers. Furthermore, an investigation comparing an on-sight climb with a secondary climb in intermediate performers (Draper et al., 2008) also found significant differences between ascents for both somatic and cognitive anxiety.

In addition to the use of questionnaires to obtain a subjective measure of psychological stress in the current study, plasma cortisol concentration was measured at specific time points in response to the climbing trial. When investigating intermediate performers, plasma cortisol concentration has been used as a physiological marker of stress in a number of rock climbing studies (Draper et al., 2008, 2010; Hodgson et al., 2009). Peak plasma cortisol concentrations were observed at $15 \mathrm{~min}$ postclimb for both top rope and lead ascents. These findings are in agreement with previous authors who suggest that peak plasma cortisol concentration occurs at 15-20 min post stressor (Smyth et al., 1998; Hruschka et al., 2005; Levine et al., 2007; Beaven et al., 2008). Figure 1 shows that peak cortisol concentration in advanced rock climbers is captured at 15 min post stress in both lead and top rope conditions. The lead climb elicited a larger cortisol response $(13.47 \mu \mathrm{g} / \mathrm{dL})$ than top rope; however, this was shown to be non significant.

In addition to the lack of significant differences between top rope and lead for the measures obtained by CSAI-2R and plasma cortisol concentrations, regression analysis revealed no significant relationships between anxieties or self-confidence and cortisol concentration. This is contrary to the findings of Draper et al. (2011b), who found significant linear relationships between plasma cortisol concentration and self-confidence $\left(r=-0.52, \quad R^{2}=0.267, \quad\right.$ and $\left.P=0.024\right), \quad$ cognitive $\left(r=0.5, R^{2}=0.253\right.$, and $\left.P=0.028\right)$ and somatic anxiety ( $r=0.46, R^{2}=0.210$, and $P=0.049$ ) in response to an on-sight ascent at the top of the participants ability.

More advanced climbers who take leader falls regularly as a consequence of training appear to have no concerns for falling indoors. Advanced climbers appear to have an understanding of perceived and real risk. The difference in these findings can therefore be attributed to the level of ability and experience of the climbers. Furthermore, it has been suggested that climbers who participate at a high level on a regular basis may have both physiological and psychological adaptations (Goddard \& Neumann, 1994; Ferguson \& Brown, 1997; Horst \& Fleming, 2010). Climbers in the current study trained more frequently (three to four times a week) than those reported by (Draper et al., 2011a) and performed at a much higher level (attaining a best on-sight grade of over 4 Ewbank). As the current study shows no significant differences or relationships in plasma cortisol concentration or CSAI-2R between the ascent styles lead and top rope, it is likely that the elevations in cortisol concentration seen in Fig. 1 are a consequence of the physiological stress of climbing and not an emotional response.

Several previous studies have reported $\mathrm{VO}_{2}$ and $\mathrm{Hr}$ responses relative to maximal values obtained during cycle ergometry or treadmill running (Billat et al., 1995; Watts \& Drobish, 1998; Sheel, 2003; de Geus et al., 2006; Bertuzzi et al., 2007). Findings from these studies have shown a disproportionate rise in $\mathrm{Hr}$ for a given $\mathrm{VO}_{2}$ value. Previously reported values for rock climbing have ranged from $42 \%$ to $51.2 \%$ and from $77 \%$ to $86.6 \%$ for $\mathrm{VO}_{2}$ and $\mathrm{Hr}$, respectively (Billat et al., 1995; Sheel, 2003; Draper et al., 2010). Findings from the current study reveal a similar breakdown in the $\mathrm{VO}_{2}$ and $\mathrm{Hr}$ relationship; however, a novel finding of this investigation was that lead climbing appears to elicit a smaller breakdown (13\% difference) in the relationship than top rope climbing (28\% difference). Although not directly measured in the current study, climbing style, technique, and the additional demand of clipping may have affected the breakdown in the $\mathrm{VO}_{2}$ and $\mathrm{Hr}$ relationship in lead climbing. To further understand the differences between lead and top rope climbing for $\mathrm{Hr}$ and $\mathrm{VO}_{2}$, Figs 2 and 3 were produced. Figure 3 shows a steep increase in $\mathrm{VO}_{2}$ 


\section{A psychophysiological comparison of advanced rock climbers}

throughout the climb until clip 4 (approximately halfway through the route) for both lead and top rope ascents. At this point, $\mathrm{VO}_{2}$ appears to plateau until the finish, suggesting a climbing steady state has occurred in both ascent styles. It has been proposed that trained individuals are able to activate their aerobic metabolism faster than nontrained athletes as well as having a smaller $\mathrm{O}_{2}$ deficit (Demarle et al., 2001). This may have caused the plateau in $\mathrm{VO}_{2}$ seen at clip 4 for both ascent styles as all participants in the current study train on a regular basis (Table 1).

Similarly, average $\mathrm{Hr}$ increased in both ascent styles at the onset of climbing. Average lead $\mathrm{Hr}$ was higher than top rope throughout the climb; however, this was found to be non significant until clip 6. The consistently higher Hr seen in Fig. 2 may be a consequence of lead climbing requiring prolonged periods of time spent in isometric contraction while clipping the safety rope, something not required in top rope climbing. It has been well documented that isometric contractions cause an elevated $\mathrm{Hr}$ response during forearm and handgrip exercise (Kilbom \& Brundin, 1976; Bystrom \& Kilbom, 1990; Bystrom et al., 1991). More recently, Osada et al. (2003) showed that during isometric handgrip exercise, the greater the contraction, the greater the hyperemic effect, which consequently increases forearm blood flow. It is therefore possible that $\mathrm{Hr}$ is elevated in lead climbing as a direct consequence of clearing increased forearm muscle metabolites. In reviewing the profile and demand of the climb, clip 6 to the finish occurred after the climbers ascended the overhung section of the wall. Here, levels of isometric contraction would have been at their greatest and most prolonged. In support of this, Osada et al. (2003) compared forearm blood flow recovery during different intensities of contraction and found that the stronger the contraction, the more delayed the recovery, suggesting that the increases in $\mathrm{Hr}$ may not be present until after the difficult crux section of the route.

Results for mean blood lactate concentration suggest that both lead and top rope groups began their ascents in similar physiological states post-warm-up. Table 2 shows postclimb values similar to those found during other studies investigating the physiological responses of advanced rock climbers (Mermier et al., 1997; Booth et al., 1999). No significant differences were found between lead and top rope climbing during any of the sampling times. Mean values are consistently higher post-lead climbing; however, these values are minimal (0.2-0.4 mmol/L). These minimal differences in blood lactate, coupled with the non significant differences found for $\mathrm{VO}_{2}$, suggest that both lead and top rope climbing require similar aerobic and anaerobic contributions. These findings contradict those of Draper et al. (2010), who found that blood lactate values were significantly higher postlead compared with top rope climbing during an on-sight context. The authors suggested that lead climbing was physically more demanding for intermediate performers; however, this does not appear to be the case in advanced level climbers.

\section{Perspectives}

Previous research into the psychophysiology of rock climbing has focused on intermediate performers (Williams et al., 1978; Draper et al., 2008, 2010; Hodgson et al., 2009). These investigations have shown that on-sight lead climbing provokes the highest level of physiological and psychological anxiety, more so than top rope climbing. It was suggested that the increased physiological responses were a direct reflection of the heightened levels of anxiety induced by on-sight lead climbing (Draper et al., 2008). Contrary to this, the current study has found no significant differences in self-confidence or somatic and cognitive anxiety between top rope and lead climbing under on-sight conditions. As a possible adaptation acquired through training and experience, it would appear that advanced climbers find the consequence of a leader fall insignificant when ascending at the upper limits of their ability. No significant differences in cortisol concentration were observed between any time points for lead and top rope. Furthermore, no significant relationships were found to exist between CSAI-2R measures and any plasma cortisol concentrations. As there were no significant differences in any psychophysiological markers of stress, a significantly increased $\mathrm{Hr}$ during the last three clipping phases can be attributed to the increased amount of time spent in isometric contraction during lead climbing. With no significant differences between lead and top rope for either $\mathrm{VO}_{2}$ or blood lactate concentration, it is proposed that relative aerobic and anaerobic contributions remain similar in advanced climbers. These findings suggest that during an on-sight attempt, advanced climbers elicit similar psychological or physiological responses during both lead and top ascents.

Key words: rock climbing, cortisol, on-sight, top rope, lead.

\section{References}

Beaven CM, Hopkins WG, Hansen KT, Wood MR, Cronin JB, Lowe TE. Dose effect of caffeine on testosterone and cortisol responses to resistance exercise. Int J Sport Nutr Exerc Metab 2008: 18: 131-141.
Bertuzzi RCM, Franchini E, Kokubun E, Kiss MAPDM. Energy system contributions in indoor rock climbing. Eur J Appl Physiol 2007: 101: 293-300.

Billat V, Palleja P, Charlaix T, Rizzardo P, Janel N. Energy specificity of rock climbing and aerobic capacity in competitive sport rock climbers. J Sports Med Phys Fitness 1995: 35: 20-24.

Booth J, Marino F, Hill C, Gwinn T. Energy cost of sport rock climbing in 


\section{Fryer et al.}

elite performers. Br J Sports Med 1999: 33: 14-18.

Bystrom SEG, Kilbom A. Physiological response in the forearm during and after isometric intermittent handgrip. Eur J Appl Physiol Occup Physiol 1990: 60: 457-466.

Bystrom SEG, Mathiassen SE, Fransson-Hall C. Physiological effects of micropauses in isometric handgrip exercise. Eur J Appl Physiol Occup Physiol 1991: 63: 405-411.

Cox RH, Martens MP, Russell WD. Measuring anxiety in athletics: the revised competitive state anxiety inventory-2. J Sport Exerc Psychol 2003: 25: 519-533.

de Geus B, Villanueva OíDriscoll S, Meeusen R. Influence of climbing style on physiological responses during indoor rock climbing on routes with the same difficulty. Eur J Appl Physiol 2006: 98: 489-496.

Demarle AP, Slawinski JJ, Laffite LP, Bocquet VG, Koralsztein JP, Billat VL. Decrease of $\mathrm{O} 2$ deficit is a potential factor in increased time to exhaustion after specific endurance training. J Appl Physiol 2001: 90: 947.

Draper N, Couceiro J, Fryer S, Dickson T, Winter D, Ellis G, Hamlin M, Shearman J, North C. Reporting climbing grades and grouping categories for rock climbng. Isokinet Exerc Sci 2011a: 19: 1-8.

Draper N, Fryer S, Dickson T, Blackwell G. Plasma cortisol concentrations and perceived anxiety in response to on-sight rock climbing. Int J Sports Med 2011b: doi http://dx-doi.org/ 10.1055/5-0031-1284348.

Draper N, Hodgson C. Adventure sport physiology. Chichester: WileyBlackwell, 2008: 169-170.

Draper N, Jones GA, Fryer S, Hodgson C, Blackwell G. Effect of an on-sight lead on the physiological and psychological responses to rock climbing. J Sports Sci Med 2008: 7: 492-498.

Draper N, Jones GA, Fryer S, Hodgson CI, Blackwell G. Physiological and psychological responses to lead and top rope climbing for intermediate rock climbers. Eur J Sport Sci 2010: 10: 13-20.

EspaÒa-Romero V, Ortega Porcel FB, Artero EG, JimÈnez-PavÛn D, GutiÈrrez Sainz A, Castillo GarzÛn MJ, Ruiz JR. Climbing time to exhaustion is a determinant of climbing performance in high-level sport climbers. Eur J Appl Physiol 2009: 107: 517-525.

Ferguson RA, Brown MD. Arterial blood pressure and forearm vascular conductance responses to sustained and rhythmic isometric exercise and arterial occlusion in trained rock climbers and untrained sedentary subjects. Eur J Appl Physiol Occup Physiol 1997: 76: 174-180.

Fryer S, Draper N, Dickson T, Blackwell G, Winter D, Ellis G. Comparison of lactate sampling sites for rock climbing. Int J Sports Med 2011: 32: 428-432.

Giles L, Rhodes E, Taunton JE. The physiology of rock climbing. Sports Med 2006: 36: 529-545.

Goddard D, Neumann U. Performance rock climbing. Mechanicsburg: Stackpolebooks, 1994: 4-12.

Hardy L, Hutchinson A. Effects of performance anxiety on effort and performance in rock climbing: a test of processing efficiency theory. Anxiety Stress Coping 2007: 20: 147-161.

Hart SG, Staveland LE. Development of NASA-TLX (Task Load Index): Results of empirical and theoretical research. In: Hancock A, Meshkati N, ed/eds. Human mental workload. Amsterdam: North Holland Press, 1988: 139-183.

Hodgson CI, Draper N, McMorris T, Jones G, Fryer S, Coleman I. Perceived anxiety and plasma cortisol concentrations following rock climbing with differing safety rope protocols. $\mathrm{Br}$ J Sports Med 2009: 43: 531.

Horst EJ, Fleming R. Maximum climbing: mental training for peak performance and optimal experience. Guilford: Globe Pequot Press, 2010: 139-178.

Hruschka DJ, Kohrt BA, Worthman CM. Estimating between-and within-individual variation in cortisol levels using multilevel models. Psychoneuroendocrinology 2005: 30: 698-714.

Kilbom A, Brundin T. Circulatory effects of isometric muscle contractions, performed separately and in combination with dynamic exercise. Eur J Appl Physiol Occup Physiol 1976: 36: 7-17.

Levine A, Zagoory-Sharon O, Feldman R, Lewis JG, Weller A. Measuring cortisol in human psychobiological studies. Physiol Behav 2007: 90: 43-53.

Lewis JG, Elder PA. An enzyme-linked immunosorbent assay (ELISA) for plasma cortisol. J Steroid Biochem 1985: 22: 673-676.

Mermier CM, Janot JM, Parker DL, Swan JG. Physiological and anthropometric determinants of sport climbing performance. Br J Sports Med 2000: 34: 359.

Mermier CM, Robergs RA, McMinn SM, Heyward VH. Energy expenditure and physiological responses during indoor rock climbing. Br J Sports Med 1997: 31: 224.

NoÈ F, Quaine F, Martin L. Influence of steep gradient supporting walls in rock climbing: biomechanical analysis. Gait Posture 2001: 13: 86-94.

Osada T, Katsumura T, Murase N, Sako T, Higuchi H, Kime R, Hamaoka T, Shimomitsu T. Post-exercise hyperemia after ischemic and non-ischemic isometric handgrip exercise. J Physiol Anthropol Appl Human Sci 2003: 22: 299-309.

Quaine F, Vigouroux L, Martin L. Effect of simulated rock climbing finger postures on force sharing among the fingers. Clin Biomech 2003: 18: 385-388.

Sanchez X, Lambert P, Jones G, Llewellyn DJ. Efficacy of pre ascent climbing route visual inspection in indoor sport climbing. Scand J Med Sci Sports 2010: doi: 10.1111/j.1600 0838.2010.01151.x.

Sheel AW. Physiology of sport rock climbing. Br J Sports Med 2004: 38: 355.

Sheel W. Physiological responses to indoor rock-climbing and their relationship to maximal cycle ergometry. Med Sci Sports Exerc 2003: 35: 1225.

Sherk VD, Sherk KA, Kim SJ, Young $\mathrm{KC}$, Bemben DA. Hormone responses to a continuous bout of rock climbing in men. Eur J Appl Physiol 2010: 111: $1-7$.

Smyth J, Ockenfels MC, Porter L, Kirschbaum C, Hellhammer DH, Stone AA. Stressors and mood measured on a momentary basis are associated with salivary cortisol secretion. Psychoneuroendocrinology 1998: 23: 353-370.

Vigouroux L, Quaine F, Labarre-Vila A, Moutet F. Estimation of finger muscle tendon tensions and pulley forces during specific sport-climbing grip techniques. J Biomech 2006: 39: 2583-2592.

Volovitz B, Kauschansky A, Nussinovitch M, Harel L, Varsano I. Normal diurnal variation in serum cortisol concentration in asthmatic children treated with inhaled budesonide. J Allergy Clin Immunol 1995: 96: 874-878.

Watts PB, Daggett M, Gallagher P, Wilkins B. Metabolic response during sport rock climbing and the effects of active versus passive recovery. Int $\mathrm{J}$ Sports Med 2000: 21: 185-190.

Watts PB, Drobish K. Physiological responses to simulated rock climbing at different angles. Med Sci Sports Exerc 1998: 30: 1118.

Williams ES, Taggart P, Carruthers M. Rock climbing: observations on heart rate and plasma catecholamine concentrations and the influence of oxprenolol. Br J Sports Med 1978: 12: 125. 